\title{
Measurement of keV-neutron capture cross sections and capture gamma-ray spectra of $\mathrm{Cs}-133$ and I-127
}

\author{
Seigo Umezawa ${ }^{a}$, Masayuki Igashira, Tatuya Katabuchi, Moraru Dominic, Shotaro Yanagida, and Tomohiro Okamiya \\ Laboratory for Advanced Nuclear Energy, Tokyo Institute of Technology, Japan
}

\begin{abstract}
The neutron capture cross sections and the capture gamma-ray spectra of ${ }^{127} \mathrm{I}$ and ${ }^{133} \mathrm{Cs}$ at incident neutron energies from 15 to $100 \mathrm{keV}$ have been measured by the time-of-flight method. Capture gammarays were detected with an anti-Compton $\mathrm{NaI}(\mathrm{Tl})$ spectrometer, and the pulse-height weighting technique was applied to derive capture yields. The capture cross sections of ${ }^{127} \mathrm{I}$ and ${ }^{133} \mathrm{Cs}$ were determined using the standard capture cross section of ${ }^{197} \mathrm{Au}$. The total errors of the cross sections were $3.8-5.1 \%$. The obtained cross sections were compared with evaluated values in JENDL-4.0 and ENDF/B-VII.1. For ${ }^{127}$ I, the energy dependence is different between the present results and the evaluations. For ${ }^{133} \mathrm{Cs}$, the evaluated values in JENDL-4.0 agree with the present results but the evaluated values in ENDF/B-VII.1 are smaller than the present results by $14 \%-18 \%$. The capture gamma-ray spectra of ${ }^{133} \mathrm{Cs}$ and ${ }^{127} \mathrm{I}$ were derived by unfolding the pulse height spectra with detector response functions.
\end{abstract}

\section{Introduction}

A key issue of the current nuclear energy production is disposal of high-level radioactive waste from nuclear power plants. Currently-planned geological disposal is controversial because long-lived nuclides existing in spent nuclear fuel require very long-term management. In order to solve the issue, researchers have suggested nuclear transmutation systems that transmute long-lived nuclides to shorter-lived or stable species via neutron-induced reactions. The systems can reduce the impact of nuclear waste disposal on the environment [1].

${ }^{129} \mathrm{I}$ and ${ }^{135} \mathrm{Cs}$ are long-lived fission products (LLFPs), which are desired to be transmuted. The cumulative fission yields of I isotopes and Cs isotopes for tharmal neutron fission of ${ }^{235} \mathrm{U}$ and ${ }^{239} \mathrm{Pu}$ are shown in Tables 1 and 2. As seen in these tables, long-lived fission products exist together with their stable isotopes in spent nuclear fuel. To design the nuclear transmutation systems of LLFPs ${ }^{129} \mathrm{I}\left(\mathrm{T}_{1 / 2}=15.7 \mathrm{M}\right.$ year $)$ and ${ }^{135} \mathrm{Cs}\left(\mathrm{T}_{1 / 2}=\right.$ $2.3 \mathrm{M}$ year), accurate neutron capture cross section data are needed not only of the LLFPs but also of the stable isotopes ${ }^{127} \mathrm{I}$ and ${ }^{133} \mathrm{Cs}$ unless isotope separation is carried out.

The neutron capture cross sections of ${ }^{127} \mathrm{I}$ and ${ }^{133} \mathrm{Cs}$ have been evaluated in nuclear data libraries based on experimental data [2-10] but the evaluated cross sections in JENDL-4.0 [11,12] and ENDF/B-VII.1 [13,14] differ by $20 \%$ at a maximum in the $\mathrm{keV}$ energy region. This motivated us to carry out the present measurements. We measured the neutron capture cross sections of ${ }^{127} \mathrm{I}$ and ${ }^{133} \mathrm{Cs}$ in the energy region from 15 to $100 \mathrm{keV}$. In addion, we measured the neutron capture gamma-ray spectra of ${ }^{127} \mathrm{I}$ and ${ }^{133} \mathrm{Cs}$, which give more information on reation mechanism of the neutron capture reaction.

\footnotetext{
a e-mail: umezawa.s.aa@m.titech.ac.jp
}

\section{Experimental procedure}

Details of the experimental procedure were reported in [15]. Only a brief description is given in this report. Experiments were performed using a $3 \mathrm{MV}$ Pelletron accelerator at the Laboratory for Advanced Nuclear Energy at the Tokyo institute of Technology. A pulsed neutron beam was produced thorough the ${ }^{7} \mathrm{Li}(\mathrm{p}, \mathrm{n})^{7} \mathrm{Be}$ reaction induced by a pulsed proton beam from the Pelletron accelerator. The pulse width of the proton beam was $1.5 \mathrm{~ns}$ and the repetition rate was $4 \mathrm{MHz}$. The proton energy was set at $1.902 \mathrm{MeV}, 22 \mathrm{keV}$ above the reaction threshold of ${ }^{7} \mathrm{Li}(\mathrm{p}, \mathrm{n})$ reaction. The time-of-flight (TOF) method was employed to deduce the incident neutron energy. Neutrons were detected with a ${ }^{6} \mathrm{Li}$ glass scintillator (scintillator size: $5 \mathrm{~mm}$ diam. $\times 5 \mathrm{~mm}$ thick) placed at a flight distance of $30 \mathrm{~cm}$. The detection angle of the ${ }^{6} \mathrm{Li}$ glass scintillator with respect to the proton beam direction was $8.7^{\circ}$, the average angle of neutrons incident on a $55 \mathrm{~mm}$-diamter sample placed at a distance $12 \mathrm{~cm}$ from the neutron source. The energy of the incident neutrons distributed from a few $\mathrm{keV}$ to $100 \mathrm{keV}$. The flight path length from the neutron source to the sample was $12 \mathrm{~cm}$.

Neutron capture gamma-rays emitted from the samples were detected with an anti-Compton $\mathrm{NaI}(\mathrm{Tl})$ spectrometer [16]. The spectrometer consists of a main $\mathrm{NaI}(\mathrm{Tl})$ detector (152 mm diam. $\times 305 \mathrm{~mm}$ length) and an annular antiCompton $\mathrm{NaI}(\mathrm{Tl})$ detector $(330 \mathrm{~mm}$ outer diam. $\times 172 \mathrm{~mm}$ inner diam. $\times 356 \mathrm{~mm}$ length) surrounding the main $\mathrm{NaI}(\mathrm{Tl})$ detector. The spectrometer was shielded with borated polyethylene, borated paraffin, potassium-free lead, cadmium, and ${ }^{6} \mathrm{LiH}$. We recorded the pulse-height $(\mathrm{PH})$ and the TOF of $\mathrm{NaI}(\mathrm{Tl})$ signals in list-data format sequentially.

Samples in chemical forms of CsI and $\mathrm{PbI}_{2}$ were used because cesium and iodine in metal form are chemically unstable in the air. In addition, the natural 
Table 1. Cumulative fission yields (\%) of I isotopes for tharmal neutron fission of ${ }^{235} \mathrm{U}$ and ${ }^{239} \mathrm{Pu}$.

\begin{tabular}{llll}
\hline & ${ }^{127} \mathrm{I}$ & ${ }^{129} \mathrm{I}$ & ${ }^{131} \mathrm{I}$ \\
\hline${ }^{235} \mathrm{U}$ & 0.1522 & 0.5388 & 2.895 \\
${ }^{239} \mathrm{Pu}$ & 5.0665 & 1.3214 & 3.8562 \\
\hline
\end{tabular}

Table 2. Cumulative fission yields (\%) of Cs isotopes for tharmal neutron fission of ${ }^{235} \mathrm{U}$ and ${ }^{239} \mathrm{Pu}$.

\begin{tabular}{cccc}
\hline & ${ }^{133} \mathrm{Cs}$ & ${ }^{135} \mathrm{Cs}$ & ${ }^{137} \mathrm{Cs}$ \\
\hline${ }^{235} \mathrm{U}$ & 6.7000 & 6.5342 & 6.1636 \\
${ }^{239} \mathrm{Pu}$ & 7.0154 & 7.6171 & 6.6038 \\
\hline
\end{tabular}

isotope abundance of both ${ }^{133} \mathrm{Cs}$ and ${ }^{127} \mathrm{I}$ is $100 \%$. To estimate contribution from lead in the $\mathrm{PbI}_{2}$ measurement, we also used a metal lead sample. ${ }^{197} \mathrm{Au}$ was also used as a standard sample for neutron capture cross section. The physical form of the $\mathrm{CsI}$ and $\mathrm{PbI}_{2}$ samples was powder. The samples were sealed in graphite containers. The diameters of the samples were $55 \mathrm{~mm}$. The weights of the samples were $10.04 \mathrm{~g}$ and $9.94 \mathrm{~g}$ for $\mathrm{CsI}$ and $\mathrm{PbI}_{2}$.

Measurements were repeated cyclically for the CsI sample (CsI run), $\mathrm{PbI}_{2}$ sample ( $\mathrm{PbI}_{2}$ run), $\mathrm{Pb}$ sample $(\mathrm{Pb}$ run), ${ }^{197} \mathrm{Au}$ sample ( ${ }^{197} \mathrm{Au}$ run) and no sample (blank run).

\section{Data analysis}

Recorded TOF-PH event data were analyzed. Four foreground and one background TOF gates were set for analysis. The corresponding energies to the four foreground gates were $15-25 \mathrm{keV}$ (Gate 1), $25-35 \mathrm{keV}$ (Gate 2), 35-55 keV (Gate 3), and 55-100 keV (Gate 4). The foreground and the background pulse-height spectra were obtained by sorting the TOF-PH event data with the TOF gates. The net pulse-height spectra were obtained by subtracting the background pulse-height spectra from the foreground pulse-height spectra.

A pulse-height weighting technique [2] was applied to derive the capture yields. The absolute cross sections were determined from standard measurements of a gold sample, based on the well-known cross section of ${ }^{197} \mathrm{Au}(\mathrm{n}, \gamma){ }^{198} \mathrm{Au}$ [17] of ENDF/B-VII.1. We also made corrections for neutron self-shielding and multiple scattering in the samples.

The neutron capture cross section of ${ }^{127}$ I was obtained by subtracting the yield contribution of $\mathrm{Pb}$ from the obtained capture yield of the $\mathrm{PbI}_{2}$ sample. Similarly, the neutron capture cross section of the ${ }^{133} \mathrm{Cs}$ was obtained by subtracting the yield contribution of ${ }^{127} \mathrm{I}$ from the obtained capture yield of CsI.

In addition to statistical errors, systematic uncertainties of the ${ }^{197} \mathrm{Au}(\mathrm{n}, \gamma){ }^{198} \mathrm{Au}$ cross section, weighting function, correction of discriminated gamma-ray contribution below $600 \mathrm{keV}$, correction of neutron self-shielding and multiple scattering, and impurity correction were included in the data analysis. The major components of the errors were statistics $(\sim 1 \%)$, the gold capture cross sections $(3 \%)$, discriminated gamma-ray corrections $(\sim 1.5 \%)$, and selfshielding and multiple scattering (1-2\%).

The capture gamma-ray spectrum was derived by unfolding the net pulse-height spectrum with detector response functions.

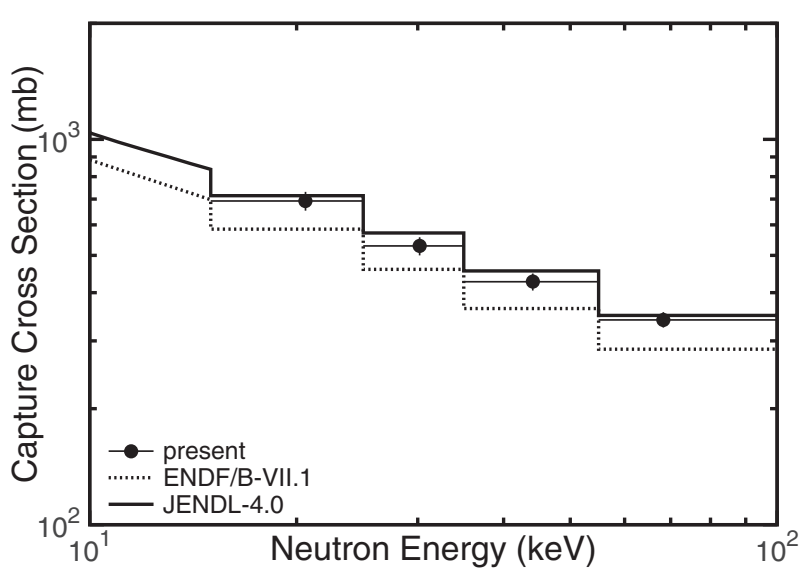

Figure 1. Neutron capture cross sections of ${ }^{127} \mathrm{I}$. The present results are shown as solid circles. Evaluated data of JENDL4.0 [11] and ENDF/B-VII.1 [13] are compared with the present results. The evaluations were averaged over the same energy bins as the present experimental data.

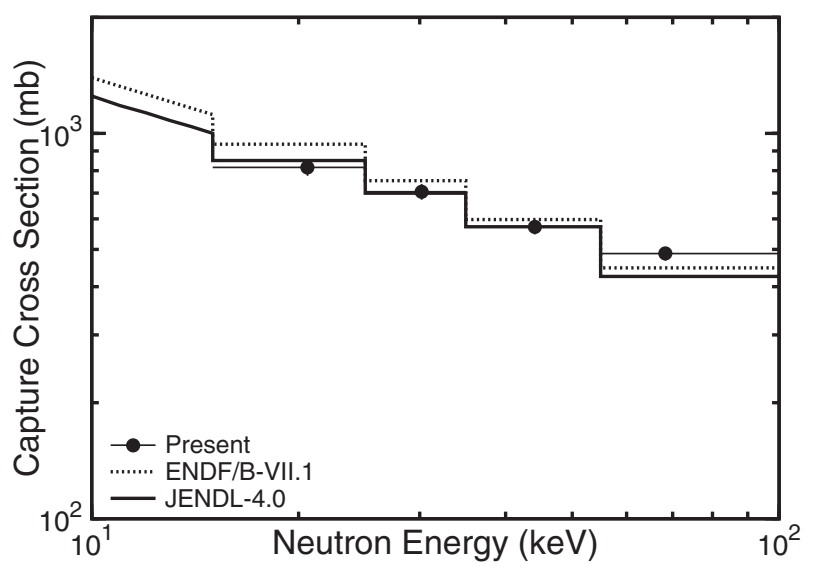

Figure 2. Neutron capture cross sections of ${ }^{133} \mathrm{Cs}$. The present results are shown as solid circles. Evaluated data of JENDL4.0 [12] and ENDF/B-VII.1 [14] are compared with the present results. The evaluations were averaged in the same energy bins as the present experimental data.

\section{Results and discussion}

\subsection{Capture cross sections}

The capture cross sections of ${ }^{127} \mathrm{I}$ and ${ }^{133} \mathrm{Cs}$ were derived with errors of about $5 \%$ in the neutron energy region from 15 to $100 \mathrm{keV}$. The present neutron capture cross sections are shown together in Figs. 1 and 2. The evaluated cross sections of JENDL-4.0 [11,12] and ENDF/B-VII.1 [13, 14] are also shown in the figures. The cross section values are averaged over the same energy bins as the present experimental data for comparison. For ${ }^{127} \mathrm{I}$, the evaluated cross sections are higher than the present results at the lowest energy (15-25 keV) and lower at the highest energy $(55-100 \mathrm{keV})$. For ${ }^{133} \mathrm{Cs}$, the evaluated cross section of JENDL-4.0 is in good agreement with the present results. On the other hand, the cross section of ENDF/B-VII.1 is lower than the present results by $14-18 \%$.

\subsection{Capture gamma-ray spectra}

The neutron capture gamma-ray spectra of ${ }^{127} \mathrm{I}$ and ${ }^{133} \mathrm{Cs}$ were derived by unfolding the net pulse height spectra with 


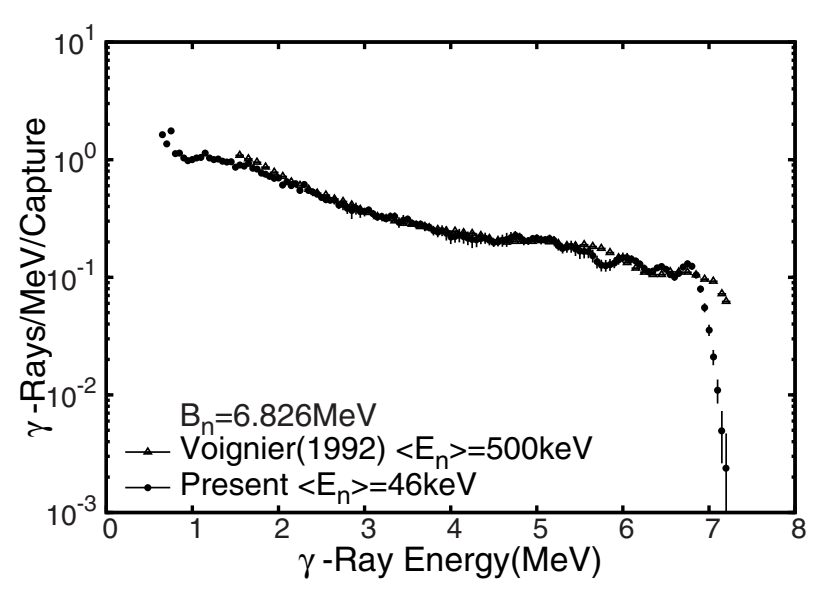

Figure 3. Capture gamma-ray spectrum of ${ }^{127} \mathrm{I}$ in the neutron energy region from 15 to $100 \mathrm{keV}$. Previous measurement [18] is compared with the present results.

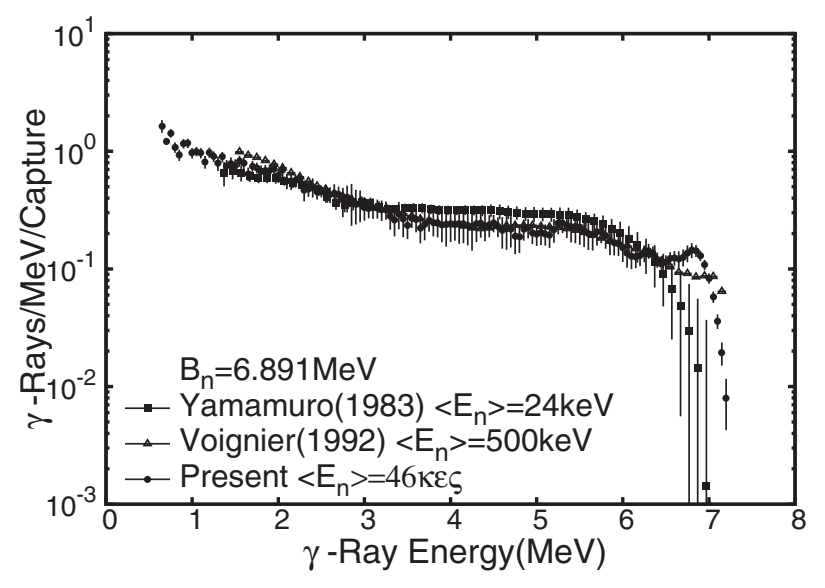

Figure 4. Capture gamma-ray spectrum of ${ }^{133} \mathrm{Cs}$ in the neutron energy region from 15 to $100 \mathrm{keV}$. Previous measurements [9], [18] are compared with the present results.

Table 3. Multiplicities of observed capture gamma-rays.

\begin{tabular}{ccc}
\hline Nuclide & $\mathrm{B}_{n}+<\mathrm{E}_{n}>[\mathrm{MeV}]$ & Multiplicties \\
\hline${ }^{127} \mathrm{I}$ & 6.872 & $2.67 \pm 0.02$ \\
${ }^{133} \mathrm{Cs}$ & 6.937 & $2.56 \pm 0.04$ \\
\hline
\end{tabular}

detector response functions, as shown in Figs. 3 and 4. The previous measurements are also shown for comparison.

Voignier et al. [18] measured the capture gamma-ray spectra of ${ }^{127} \mathrm{I}$ and ${ }^{133} \mathrm{Cs}$ at a neutron energy of $500 \mathrm{keV}$. Yamamuro et al. [9] measured the capture gamma-ray spectra of ${ }^{133} \mathrm{Cs}$ in the neutron energy $24 \mathrm{keV}$. Except for difference of high energy endpoint due to the neutron energy difference, overall shape of the spectra agrees between the previous and measurements.

The gamma-ray multiplicities $\left(\mathrm{E}_{\gamma} \geq 0.65 \mathrm{MeV}\right)$ calculated from the gamma-ray spectra are shown in Table 3.

\section{Conclusions}

The neutron capture cross sections of ${ }^{127} \mathrm{I}$ and ${ }^{133} \mathrm{Cs}$ were measured with the errors of $3.8-5.1 \%$ at incident neutron energies from 15 to $100 \mathrm{keV}$.

Comparison with evaluated cross sections of JENDL4.0 and ENDF/B-VII.1 shows that the present results of
${ }^{127} \mathrm{I}$ and the evaluations are in almost good agreement but have slightly different energy dependence.

In comparison of the capture cross section of ${ }^{133} \mathrm{Cs}$, the JENDL-4.0 evaluation agrees with the present results but the ENDF/ B-VII.1 evaluation is by $14-18 \%$ lower than the present results.

The capture gamma-ray spectra of ${ }^{133} \mathrm{Cs}$ and ${ }^{127} \mathrm{I}$ were derived by unfolding the net pulse-height spectra. The gamma-ray multiplicities $\left(\mathrm{E}_{\gamma} \geq 0.65 \mathrm{MeV}\right)$ were obtained from the capture gamma-ray spectra. Capture gamma-ray spectra give more information to determine parameters of nuclear reaction model calculations. The present gammaray spectra will be used in future theoretical work.

\section{References}

[1] H. Takano, T. Ikegami, "Activities on R \& D of partitioning and transmutation in Japan". Actinide and Fission Product Partitioning and Transmutation Seventh Information Exchange Mtg., Jeju, Republic of Korea, Oct. 14-16, 2002, 23-35 (2003)

[2] R.L. Macklin, J.H. Gibbons "capture-cross-section studies for $30-220 \mathrm{keV}$ neutrons using a new technique", Phys. Rev. 159, 1007-1012 (1967)

[3] N. Yamamuro, T. Doi, T. Miyagawa, Y. Fujita, K. Kobayashi, R.C. Block, "Measurement of Neutron Capture Cross Sections with Fe-Filtered Beam" J. Nucl. Sci. Technol. 15(9), 637-644 (1978)

[4] N. Yamamuro, K. Saito, T. Emoto, T. Wada, T. Fujita, K. Kobayashi, "Neutron Capture Cross Section Measurements of Nb-93, I-127, Ho-165, Ta-181 and U-238 between 3.2 and $80 \mathrm{keV}$ " J. Nucl. Sci. Technol. 17(8), 582-592 (1980)

[5] R.L. Macklin, "Neutron Capture Cross Sections and Resonances of Iodine-127 and Iodine-129" Nucl. Sci. and Eng. 85(4), 350-361 (1983)

[6] G. Noguere, O. Bouland, A. Brusegan, P.Schillebeeeckx, P. Siegler, A. Lepretre, N. Herault, G. Rudolf, "Neutron capture and total cross sections of ${ }^{127}$ I and ${ }^{129}$ I" Phys. Rev. C. 74, (2006)

[7] N. Yamamuro, T. Doi, T. Miyagawa, Y. Fujita, K. Kobayashi, R.C. Block, "Measurement of Neutron Capture Cross Sections with Fe-Filtered Beam" J. Nucl. Sci. Technol. 15(9), 637-644 (1978)

[8] R.L. Macklin, "Cesium-133 Neutron Capture Cross Section" Nucl. Sci. and Eng. 81, 418-422 (1982)

[9] N. Yamamuro, M. Igashira, T. Sekiya, H. Shirayanagi, "KeV-Neutron Capture in Cesium-133, Gold-197 and Tantalum-181" J. Nucl. Sci. Technol. 20(10), 797-811 (1983)

[10] M.V. Bokhovko, V.N. Kononov, N.S. Rabotnov, A.A. Voevodskiy, "Neutron radiation cross-section, neutron transmission and average resonance parametersnfor some fission product nuclei” Fiz. Energ. Institut., Obnisk Reports, 2168 (1991)

[11] JENDL-4.0 data file for 127I (MAT=5325), evaluated by JNDC FP Nuclear data W. G. (2009)

[12] JENDL-4.0 data file for 133Cs (MAT=5525), evaluated by N. Iwamoto (2010)

[13] ENDF/B-VII.1 data file for 127I (MAT=5325), evaluated by Young (2011)

[14] ENDF/B-VII.1 data file for 133Cs (MAT=5525), evaluated by Mughabghab (2011) 
[15] S. Mizuno, M. Igashira, K. Masuda, "Measurements of keV-neutron capture cross sections and capture gamma-ray spectra of 161,162,163Dy", J. Nucl. Sci. Technol. 36, 493-507 (1999)

[16] M. Igashira, H. Kitazawa, N. Yamamuro, heavy shield for the gamma-ray detector used in fastneutron experimentNucl. Instrum. Methods A 245, 432-437 (1986)
[17] ENDF/B-VII.1 data file for 197Au (MAT=7925), evaluated by P.G. Young (1991)

[18] J. Voignier, S. Joly, G. Grenier, "Capture cross sections and Gamma-ray spectra from the interaction of $0.5 \mathrm{MeV}$ to $3.0 \mathrm{MEV}$ Neutrons with Nuclei in the mass range A $=45$ to 238 " Nucl. Sci. and Eng. 112(1), 87-94 (1992) 